\title{
Timing of onset and rate of decline in learning and retention in the pre-dementia phase of Alzheimer's disease
}

\author{
Ellen Grober ${ }^{1 *}$, Yang $\mathrm{An}^{2}$, Richard B. Lipton ${ }^{1}$, Claudia Kawas ${ }^{3}$ and Susan M. Resnick ${ }^{2}$ \\ ${ }^{1}$ Department of Neurology, Albert Einstein College of Medicine and Montefiore Medical Center, Bronx, Bronx, NY 10461, USA \\ ${ }^{2}$ Laboratory of Behavioral Neuroscience, National Institute on Aging, Baltimore, MD 21224, USA \\ ${ }^{3}$ Department of Neurology, University of California Irvine, CA 92697, USA
}

(Received July 11, 2018; Final Revision February 24, 2019; Accepted March 5, 2019; First Published Online May 16, 2019)

\begin{abstract}
Objective: To examine trajectories of declines in learning and retention during the predementia phase of Alzheimer's disease (AD) using the picture version of the Free and Cued Selective Reminding Test with Immediate Recall (pFCSRT+IR). Method: Learning was defined by the sum of free recall over three test trials. Retention was defined in two ways: by delayed free recall (DFR) and by savings; DFR adjusted for learning. The performances of 217 incident AD cases from the Baltimore Longitudinal Study of Aging (BLSA) were aligned based on the time that AD was first diagnosed. The predementia phase of learning and retention decline was assessed using change point models in which cognitive trajectories are described by a series of linear components with knots delineating times of accelerating decline. Results: Trajectories for both learning and DFR had two change points: the first at 6.58 (95\% confidence intervals (CI): $6.56,6.60)$ to 7.29 (95\% CI: $6.13,8.46)$ years before diagnosis followed by gradual decline over the next 4 years, and a second acceleration of decline $1.89(0.56,3.24)$ to 2.93 (95\% CI: $1.56,4.30)$ years before diagnosis. The change points for DFR were not significantly earlier in the predementia phase than the change points for learning. Savings had one change point, 5.3 (95\% CI: 3.56, 7.04) years before diagnosis. Conclusion: Both learning and DFR showed similar profiles of decline in the years prior to the clinical diagnosis of AD. When delayed recall was adjusted for initial learning, the measure was less sensitive to early disease.
\end{abstract}

Keywords: Alzheimer's disease, prospective studies, verbal learning, retention, memory disorders, preclinical dementia, free and cued selective reminding test

\section{INTRODUCTION}

Ever since delayed recall was shown to be more sensitive than initial learning for diagnosing dementia (Welsh et al., 1991), the conventional practice has been to rely on retention measures rather than learning measures for identifying mild cognitive impairment (MCI) and dementia. This practice was challenged by an analysis of MCI participants from the Alzheimer's Disease Neuroimaging Initiative (ADNI) cohort grouped according to their learning and retention scores on the Rey Auditory Verbal Learning Test (Chang et al., 2010). Though retention is typically measured using delayed free recall (DFR), in these analyses, DFR was adjusted for the amount of initial learning. Participants with impairments on both learning and retention measures showed the highest conversion rate to clinical dementia over 2 years. Furthermore, participants with learning deficits regardless of retention level

*Correspondence and reprint requests to: Ellen Grober, Department of Neurology, Albert Einstein College of Medicine and Montefiore Medical Center, Bronx, Bronx, NY, USA. E-mail: ellen.grober@einstein.yu.edu showed a higher conversion rate than those with retention deficits regardless of learning level. Relying on retention measures alone, therefore, may miss an important subset of older adults at risk for developing Alzheimer's disease (AD) (Chang et al., 2010). Comparing predictive validity is one approach to identifying measures sensitive to early $\mathrm{AD}$.

Change point methods provide another approach for identifying measures that signal cognitive decline in the predementia phase of $\mathrm{AD}$. These models align participants at the time of dementia diagnosis and look backwards in time to describe prediagnostic cognitive trajectories using a series of piece-wise linear components separated by knots (change points). These change points delineate times of accelerating decline in the predementia phase of AD (Hall et al., 2001, 2003). This approach was applied to a previous sample from the Baltimore Longitudinal Study of Aging (BLSA) (Grober et al., 2008) in which learning was defined by the sum of free recall (SumFR) on the picture version of the Free and Cued Selective Reminding Test with Immediate Recall (pFCSRT+IR) (Buschke, 1984; Grober \& Buschke, 1987). 
Seven years before clinical dementia diagnosis, there was an accelerated decline in SumFR in 92 incident AD cases that developed from 1985 to 2000 with no detectable free recall decline before then (Grober et al., 2008). After this first change point, there was a decline of 1.48 items per year (out of 48) that continued until a second acceleration 2.6 years before dementia diagnosis when the rate of decline doubled.

Retention was also assessed using the pFCSRT + IR in a case-control study of BLSA participants (Grober \& Kawas, 1997). Learning was defined by the SumFR. Retention was a savings index defined by DFR divided by third trial free recall. Twenty incident AD cases displayed impaired learning but intact retention relative to 60 matched controls at baseline. Three years later, retention was impaired and learning had deteriorated further (Grober \& Kawas, 1997).

Administration of the pFCSRT+IR has continued in the BLSA, and the number of preclinical AD cases that have developed dementia has increased from 92 to 217 since our previous publication. In the present study, using this expanded data set, we describe and contrast the trajectories of decline in learning and retention during the preclinical onset of $\mathrm{AD}$ using change point models. We compared change points and slopes for measures of learning and retention in the predementia phase. Learning was measured by the SumFR over the three test trials $(\max =48)$. Retention was measured in two ways: by DFR tested approximately 15-20 min later and by the savings index.

There were three objectives. The first was to extend our earlier findings on the trajectories of learning, as measured by SumFR, during the predementia phase of $\mathrm{AD}$ using change point models (Grober et al., 2008). The second objective was to characterize the trajectories of retention, as measured by DFR, during the predementia phase of $\mathrm{AD}$. If DFR was more sensitive to early disease, we predicted that its change point would occur earlier in the course of preclinical AD; under this hypothesis, we would expect accelerated decline in DFR would begin more years prior to dementia diagnosis than the accelerated decline in SumFR. Alternatively, if SumFR was more sensitive to early disease, it should accelerate first, with a longer interval from acceleration to dementia diagnosis than shown for DFR. The final objective was to examine the trajectory of the savings index. In prior work, when retention was measured by savings, incident $\mathrm{AD}$ cases displayed intact retention 3 years before clinical diagnosis (Grober \& Kawas, 1997).

\section{METHODS}

\section{Participants}

The analyses were based on data from 217 BLSA participants who developed clinical AD between January 1985 and December 2015 and underwent longitudinal assessments with the pFCSRT+IR. All available visits meeting these requirements were included in the analysis, including data acquired after the onset of AD symptoms. The BLSA study is approved by the local institutional review board, and all participants gave written informed consent before each assessment.

\section{Dementia Diagnosis}

Clinical and neuropsychological data from each participant were reviewed at a consensus case conference if their Clinical Dementia Rating Score (CDR) was greater or equal 0.5 or if they had more than three errors on the Blessed Information-Memory-Concentration Test (BIMC: Blessed et al., 1968). Participants in the autopsy study (about half) were also evaluated by case conference upon death or withdrawal. Diagnoses of dementia and clinical AD were based on criteria outlined in the Diagnostic and Statistical Manual of Mental Disorders, third edition, revised (American Psychiatric Association, 1987) and the National Institute of Neurological and Communication Disorders and Stroke - AD and related disorders (McKhann et al., 1984). The diagnosis of dementia relied on clinical history, informant report, and a broad battery of neurocognitive tests that included pFCSRT + IR scores.

\section{pFCSRT + IR}

Before the pFCSRT+IR was administered, the 16-line drawings used in the test were presented for naming. The study phase followed in which participants were asked to search a card containing four of the drawings (e.g., grapes) for an item that goes with a unique category cue (e.g., fruit). After all four items were identified, immediate recall of just those four items was tested by free recall, followed by cued recall for missed items. When cued recall failed, the participant was told the name of the item. The study phase was repeated for all 16 drawings. The test phase consisted of three trials of free recall each followed by cued recall for items not retrieved by free recall. The sumFR was the learning measure (maximum $=48$ ). There were two retention measures: DFR tested 15-20 min after learning without representation of the items (maximum $=16$ ) and savings defined by DFR divided by third trial free recall.

\section{Statistical Analyses}

To determine the number and timing of change points, three mixed effect models with increasing complexity were fit to the data with sumFR, DFR, and the savings measures as the separate outcomes, and the time (years) to diagnosis of $\mathrm{AD}$ was the main predictor. The three models are: (1) Nochange point model, (2) one-change point model, and (3) two-change point model. The models were estimated using maximum likelihoodmethod. Model selections were based on likelihood ratio test and Akaike information criterion (AIC) (Burnham \& Anderson, 2002). The best fitting model indicates how many change points (if any) are there and estimates the timing of the change points and longitudinal trajectories at each stage. 
The two-change point model function is given by

$$
\begin{aligned}
Y_{i j}= & \beta_{0}+b_{0 i}+\beta_{1} t_{i m e_{i j}}+\beta_{2}\left(t i m e_{i j}-c_{1}\right)_{+} \\
& +\beta_{3}\left(t i m e_{i j}-c_{2}\right)_{+}+\varepsilon_{i j}
\end{aligned}
$$

where $(x)_{+}=x, x>0$ and $(x)_{+}=0, x<0$.

$c_{1}$ is the first change point and $c_{2}$ is the second change point. $b_{0 i}$ is a random effect that follows a normal distribution with mean 0 and standard deviation of $\sigma$.

To test if the timings of the change points are statistically different between learning and retention, we bootstrapped the final change point models on 500 random samples.

All the analyses were conducted in SAS 9.4 (Cary, NC).

\section{RESULTS}

The sample that developed clinical $\mathrm{AD}$ had a mean age at baseline of $75.3(S D=7.6)$ and was $49.3 \%$ women. The cohort had up to $19 \mathrm{pFCSRT}+\mathrm{IR}$ assessments with an average of $5.6(S D=3.6)$ assessments and up to 23 years of longitudinal follow-up with an average of 8.8 ( $S D=$ 6.0) years. Mean follow-up from baseline to the development of dementia was 10.4 years $(S D=6.6)$. The cohort is described in Table 1.

Table 2 shows the model fit statistics, including included AIC and likelihood ratio tests. These indices and tests show that the two-change point model fits the data best for learning and DFR and the one-change point model for savings.

For learning, the first change point is 6.58 years $(95 \%$ confidence intervals $(\mathrm{CI})=6.56,6.60$ ) before diagnosis, and the second change point is 1.89 years $(95 \% \mathrm{CI}=0.54,3.24)$ before diagnosis. The two change points result in three segments of trajectories whose rates of decline are -0.14 per year ( $p=.0017$ ) before the first change point, -1.54 per year $(p<.0001)$ between the first and second change points, and -2.50 per year $(p<.0001)$ after the second change point. At the first change point, sumFR is 31.2 and at the second it is 24.0. Figure 1 shows the model fit trajectory for learning with 95\% CI around the two change points.

For DFR, the first change point is 7.29 years $(95 \% \mathrm{CI}=$ $6.13,8.46$ ) before the diagnosis and the second change point is 2.93 years $(95 \% \mathrm{CI}=1.56,4.30)$. The two change points result in three segments of trajectories whose rates of decline are -0.031 per year $(p=.24),-.56$ per year $(p<.0001)$, and -1.06 per year $(p<.0001)$, respectively. At the first change point, DFR is 11.7 and at the second it is 9.2. Figure 2 shows the model fit trajectory for DFR with $95 \%$ CI around the two change points.

The bootstrapping results showed that the first and second change points for DFR did not differ statistically from those for learning ( $p=.38$ and .30 , respectively). Approximately 4 years separated the first and second change points in both the learning and retention trajectories.

There was only one change point for the savings index 5.30 years before diagnosis (95\% CI: 3.56, 7.04) (Figure 3). The change point resulted in two segments whose rates
Table 1. Baseline demographics and sample characteristics for persons who developed incident AD in the BLSA

\begin{tabular}{lc}
\hline \hline$N$ subjects & 217 \\
$N$ data points & 1213 \\
Male (\%) & $110(50.7)$ \\
White (\%) & $194(89.4)$ \\
Baseline age & $75.3(7.6)$ \\
& $58.5-95.4$ \\
No. of assessments & $5.6(3.6)$ \\
Follow-up relative to AD diagnosis (years) & $1-19$ \\
Age at AD diagnosis & $10.4(6.6)$ \\
& $85.6(7.0)$ \\
Education & $62-104$ \\
BMI & $16.6(2.7)$ \\
Smoking status, no. (\%) & $25.3(3.9)$ \\
Never & \\
Former & $83(38.2)$ \\
Current & $121(55.8)$ \\
Hypertension, no. (\%) & $13(6.0)$ \\
No & \\
Yes & $149(68.7)$ \\
Diabetes, no. (\%) & $68(31.3)$ \\
No & \\
Yes & $202(93.1)$ \\
Prior cardiovasc. disease ${ }^{a}$, no. $(\%)$ & $15(6.9)$ \\
No & \\
Yes & $203(93.6)$ \\
BMSE & $14(6.4)$ \\
pFCSRT+IR SumFR at learning & $27.7(2.3)$ \\
pFCSRT+IR DFR at retention & $2.5(3.3)$ \\
\hline \hline
\end{tabular}

${ }^{\text {a }}$ Defined as myocardial infarction or congestive heart failure.

of decline were $0.0042(p=.15)$ before and -0.035 ( $\mathrm{p}<.0001)$ after the change point.

\section{DISCUSSION}

Our goal was to compare the temporal unfolding of learning and retention deficits in the predementia phase of AD. The trajectories of learning (SumFR) and retention (DFR) displayed by 217 incident AD cases from the BLSA were similar: for each measure, there are two change points: the first at 6.6-7.3 years before diagnosis, followed by accelerated decline over the next 4 years. Beginning 1.9-2.9 years prior to diagnosis, there was an incremental acceleration of decline for indices of both learning and retention. Though the change points for DFR occurred earlier than SumFR, the differences were not significant. The time between the first and second change points was 4 years for both learning and retention.

The trajectory of learning deficits in the current cohort replicates and extends the findings from the earlier BLSA incident AD cohort (Grober et al., 2008). The timing of the two change points for the SumFR and the score at each point in that study were used to define the stages of objective memory 
Table 2. Model fit statistics

\begin{tabular}{|c|c|c|c|c|c|c|c|c|c|}
\hline & \multicolumn{3}{|c|}{ Learning } & \multicolumn{3}{|c|}{ DFR } & \multicolumn{3}{|c|}{ Savings } \\
\hline & AIC & $\begin{array}{l}-2 \mathrm{LL} \\
\mathrm{DF}\end{array}$ & p-value ${ }^{a}$ & AIC & $\begin{array}{l}-2 \mathrm{LL} \\
\mathrm{DF}\end{array}$ & $p$-value & AIC & $\begin{array}{c}-2 \mathrm{LL} \\
\mathrm{DF}\end{array}$ & $p$-value \\
\hline $\begin{array}{l}\text { No change } \\
\text { point }\end{array}$ & 6953.0 & $\begin{array}{c}6945.0 \\
4\end{array}$ & & 5355.9 & $\begin{array}{c}5347.9 \\
4\end{array}$ & & 659.2 & $\begin{array}{c}651.2 \\
4\end{array}$ & \\
\hline $\begin{array}{l}1 \text { change } \\
\text { point }\end{array}$ & 6604.3 & $\begin{array}{c}6592.3 \\
6\end{array}$ & $<.0001$ & 5065.1 & $\begin{array}{c}5053.1 \\
6\end{array}$ & $<.0001$ & 629.2 & $\begin{array}{c}617.2 \\
6\end{array}$ & $<.0001$ \\
\hline $\begin{array}{l}2 \text { change } \\
\text { point }\end{array}$ & 6600.4 & $\begin{array}{c}6584.4 \\
8\end{array}$ & .019 & 5054.4 & $\begin{array}{c}5038.4 \\
8\end{array}$ & .0006 & 628.4 & $\begin{array}{c}612.4 \\
8\end{array}$ & .091 \\
\hline
\end{tabular}

$\mathrm{AIC}=$ Akaike information criterion; $-2 \mathrm{LL}=-2 \log$ likelihood; $\mathrm{DF}=$ degree of freedom.

${ }^{a} p$-value is from likelihood-ratio test comparing the current model with the previous model, significance means that the current model is significantly better than the previous model.

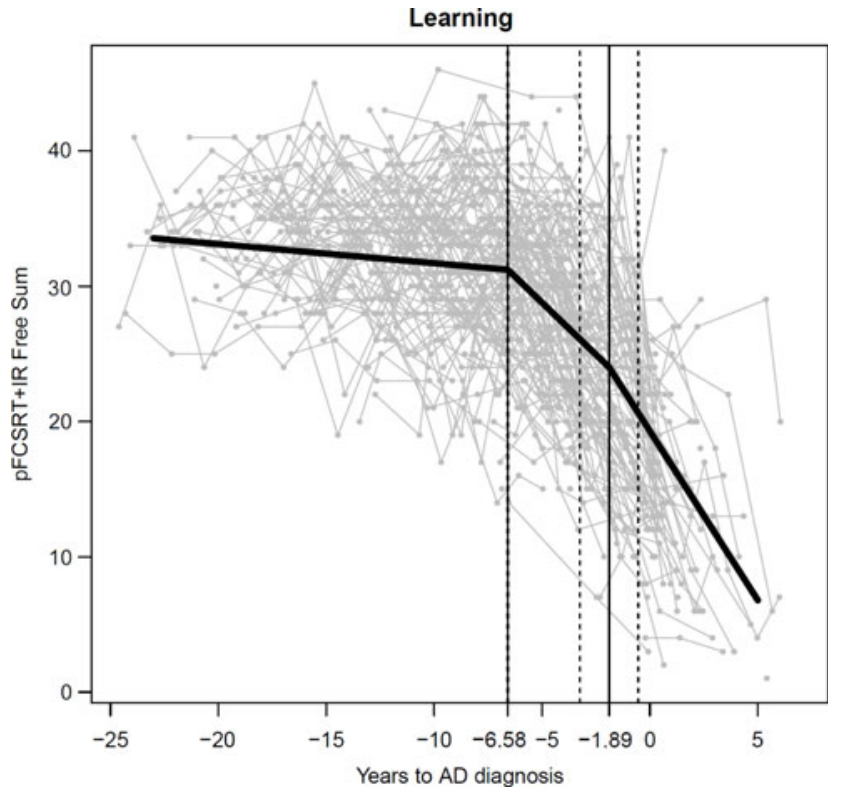

Fig. 1. Learning trajectory

impairment (SOMI) system that identifies transitional stages in the emergence of episodic memory impairment in the predementia phases of AD (Grober et al., 2018). SOMI was developed on the literature mapping FCSRT performance to clinical outcomes and biological markers, and on profiles of decline in free and total recall in longitudinal studies following cognitively normal older adults over many years, until some develop AD and other dementias. The SOMI system includes four sequential predementia stages and one clinical stage. The SOMI identified incipient dementia with excellent sensitivity and specificity (>90\%). Consistent with SOMI model predictions, time to diagnosis in the incident $\mathrm{AD}$ group was 7 years when learning was intact ( $\operatorname{sumFR}>30$ ).

The decline of learning years before clinical diagnosis is consistent with recent studies demonstrating that pFCSRT + IR may be particularly useful in secondary prevention trials aimed at reducing the progression of clinical symptoms in clinically normal $(\mathrm{CN})$ individuals with biomarker evidence of $\mathrm{AD}$. In one study, out of nine neuropsychological

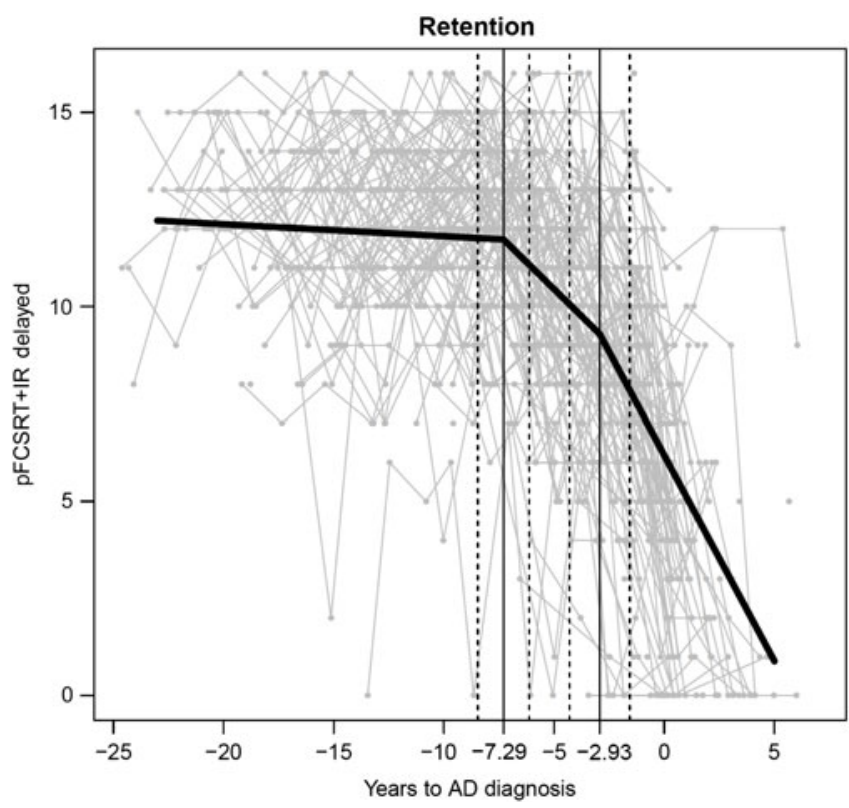

Fig. 2. Retension trajectory

tests, learning (sumFR) on the pFCSRT+IR was the only measure to demonstrate impairment at baseline in cognitively normal individuals $(\mathrm{CDR}=0)$ with the Cerebral Spinal Fluid (CSF) AD profile (Schindler et al., 2017). Further evidence that impaired learning is an early signal comes from studies of the preclinical Alzheimer cognitive composite (PACC) that includes the pFCSRT+IR (Donohue et al., 2014; Mormino et al., 2017; Papp et al., 2017). The other PACC components include delayed story recall, a timed measure of executive function (DSST), and a measure global cognition (MMSE). $\mathrm{CN}$ individuals were divided into two groups on the basis of amyloid imaging (Mormino et al., 2017). $\mathrm{A} \beta$-related change in each PACC component and the impact of adding or eliminating components were assessed. Examining effect sizes across all PACC combinations revealed that all combinations including free recall resulted in larger effect sizes over 3 and 5 years of follow-up. To determine whether progression contributed to $\mathrm{A} \beta$-related PACC decline, $A \beta+$ group was divided into those that progressed 


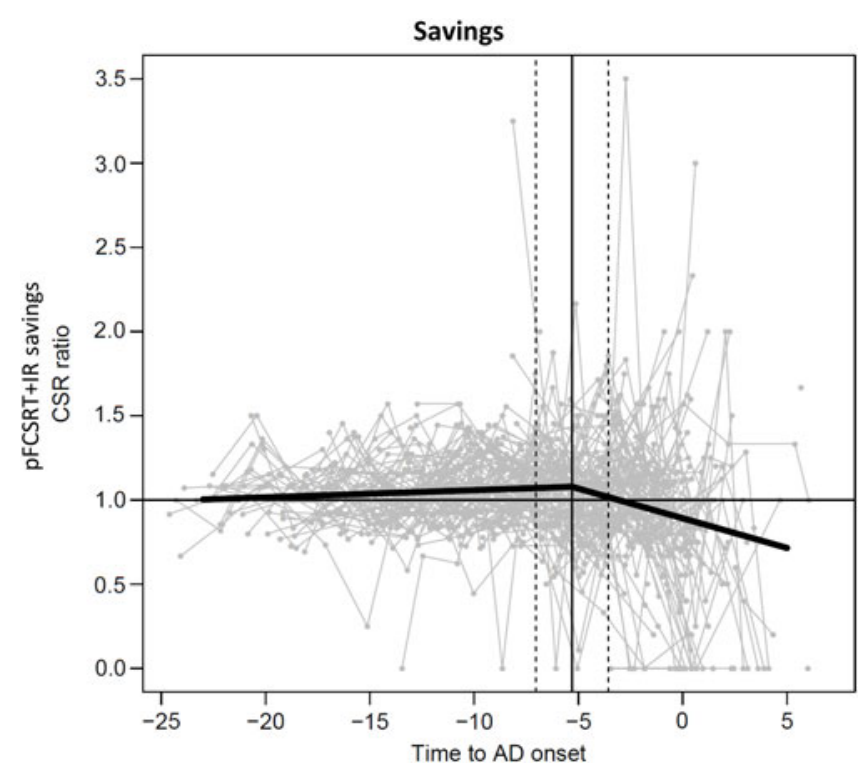

Fig. 3. Savings trajectory

to CDR 0.5 versus those that remained stable. Free recall was the only individual component to show differences between these two groups at baseline. Furthermore, the $A \beta+$ stable group did not differ from the $\mathrm{A} \beta-$ group across any PACC combinations or individual PACC components except for free recall.

For the savings index, there was only one change point, occurring 5.3 years before diagnosis, making it less sensitive to early disease than DFR. The difference in their trajectories is interesting but one that requires further investigation in the context of a larger sample including cognitively normal controls and multiple measures on the pFCSRT+IR. Except for our earlier study (Grober \& Kawas, 1997), we are not aware of any studies that examined savings using the FCSRT.

Various patterns of learning and retention decline in predementia $\mathrm{AD}$ cohorts have been observed. When learning and retention were studied using the California Verbal Learning Test in a BLSA cohort similar to the current one (Bilgel et al., 2014), learning declined before DFR among cognitively normal participants and those who progressed to $\mathrm{MCI}$ or $\mathrm{AD}$. However, delayed recall declined more rapidly than learning as the disease progressed, crossing the threshold for impairment before learning.

Using the Consortium to Establish a Registry for Alzheimer's Disease list learning test 8 years before MCI was diagnosed, impaired retention was present while impaired learning was identified 4 years later (Mistridis et al., 2015). When immediate and delayed story recall were assessed, accelerated decline of both began about 3 years prior to MCI diagnosis and rates of decline did not differ by measure (Howieson et al., 2008). Finally, amnestic MCI progressors displayed a gradual decline in learning on the word version of the FCSRT+IR 4 years before the diagnosis of $\mathrm{AD}$ dementia; decline in DFR, though starting at the same time, accelerated 1 year before diagnosis (Cloutier et al., 2015). These inconsistencies are not surprising when the factors that determine predictive value are considered: where an individual is in the multiyear process of cognitive decline that precedes dementia (Bilgel et al., 2014); the psychometric properties of the particular test being used (Grober et al., 2009); and the composition of the sample that does not go on to develop dementia.

Patterns of decline on measures of learning and retention are likely test dependent. For the pFCSRT $+\mathrm{IR}$, in contrast with some other tests, learning and retention do not differ in their sensitivity for detecting accelerated decline. Perhaps this is because the pFCSRT+IR uses controlled learning (searching for items based on category cues) which ensures semantic processing of the to-be-remembered items. Cognitive control during the study phase promotes robust learning during the test phase. Measuring retention of inadequately learned material because the learning conditions are uncontrolled adds another factor contributing to contradictory results in the pattern of learning and retention decline in predementia phase of $\mathrm{AD}$.

There are several study limitations. The same pFCSRT + IR list of items has been administered at the biennial assessments. A sensitivity analysis was conducted to assess whether practice effects due to use of the same list influenced when change points occur and rate of decline. The model included an additional practice effect term presumed to occur between the first and second assessment where the greatest practice effect occurs, though we recognize that practice effects can continue in subsequent assessments. The results show that while the practice effect is statistically significant for both learning and DFR, the main results are not materially different from the original results in terms of the number and timing of the change points. We acknowledge that this approach does not fully control for practice effects which might be expected to improve performance and reduce estimates of trajectories of decline.

Another study limitation is that $\mathrm{pFCSRT}+\mathrm{IR}$ scores were used in diagnostic case conferences through 2010 which relied on clinical history, informant report, and a broad battery of neurocognitive tests. While this raises the possibility that the diagnostic procedures influenced the timing of the change points, this seems unlikely since the first change points occurred about 7 years before participants met clinical criteria for dementia and the second change point occurred almost 2 years prior to diagnosis. Importantly, in the previous BLSA sample of incident AD cases (Grober et al., 2008), dementia diagnosis was determined independently of pFCSRT+IR scores, yet the timing and the corresponding score at each change point were similar to those reported in the current study.

Another limitation is the high-educational level of the cohort. Consistent with the cognitive reserve hypothesis (Stern, 2002), some studies have found that persons with greater education experienced accelerated memory decline closer to the time of dementia diagnosis than persons with lower education (Hall et al., 2007; Soldan et al., 2017). Thus, caution should be exercised when generalizing the temporal trajectories of learning and retention in our study 
to a less-educated cohort. The strength of our data set is the sizable and well-characterized cohort of incident AD cases and the large number of assessments available over more than 20 years of follow-up.

We have described trajectories of both learning and retention on a sample level. A point worth noting is that there were considerable variabilities in these trajectories at the individual level. The investigation of these variabilities and their potential predictors, though beyond the scope of this manuscript, is important and may shed light on the prognostic value of the measures on an individual level.

Is it necessary to include a retention measure in observational cohort studies and in interventional trials? If the study purpose is to map learning and retention processes onto the brain substrates that support them, then both measures should be collected as their impairment is associated with distinct patterns of regional brain atrophy (Chang et al., 2010). However, adding a retention test to a neuropsychological battery increases participant burden and limits the testing performed between learning and retention to nonverbal material so as not to contaminate the retention measure. Moreover, retention is a less-reliable measure than learning $(\max =16$ vs. 48). For identifying persons at high risk of $\mathrm{AD}$, impaired learning on the pFCSRT $+\mathrm{IR}$ outperformed delayed recall on both logical memory and the CERAD list learning (Wagner et al., 2012). Ultimately, the decision to include a retention measure will depend on the goals of the study.

In conclusion, we have shown that both learning and retention decline years before the onset of clinical symptoms of $\mathrm{AD}$.

\section{Acknowledgments}

The FCSRT+IR is copyrighted by the Albert Einstein College of Medicine and is made freely available for noncommercial purposes. Dr. Ellen Grober receives a small percentage of any royalties on the FCSRT+IR when it is used for commercial purposes. Dr. Yang An has no disclosures other than being an employee of the NIA. Dr. Susan Resnick has no disclosures other than being an employee of the NIA. Dr. Claudia Kawas has no disclosures. Dr. Richard B. Lipton is the Edwin S. Lowe Professor of Neurology at the Albert Einstein College of Medicine in New York. He receives research support from the NIH: 2PO1 AG003949 (Program Director), 5U10 NS077308 (PI), RO1 NS082432 (Investigator), 1RF1 AG057531 (Site PI), RF1 AG054548 (Investigator), 1RO1 AG048642 (Investigator), R56 AG057548 (Investigator), K23 NS09610 (Mentor), K23AG049466 (Mentor), and 1K01AG054700 (Mentor). $\mathrm{He}$ also receives support from the Migraine Research Foundation and the National Headache Foundation. He serves on the editorial board of Neurology, as a senior advisor to Headache, and as an associate editor to Cephalalgia. He has reviewed for the NIA and NINDS, holds stock options in eNeura Therapeutics and Biohaven Holdings; serves as consultant, advisory board member, or has received honoraria from American Academy of Neurology, Alder, Allergan, American Headache Society, Amgen, Autonomic Technologies, Avanir, Biohaven, Biovision, Boston Scientific, Dr. Reddy's, Electrocore, Eli Lilly, eNeura Therapeutics, GlaxoSmithKline, Merck, Pernix, Pfizer, Supernus, Teva, Trigemina, Vector, and Vedanta. He receives royalties from Wolff's Headache 7th and 8th Edition, Oxford Press University, 2009, Wiley and Informa. This study was supported in part by the Intramural Research Program, National Institute on Aging, NIH and the Einstein Aging Study, National Institutes of Health (AG03949), NIH.

\section{REFERENCES}

American Psychiatric Association. (1987). Diagnostic and Statistical Manual of Mental Disorders (3rd ed.). Washington, DC: American Psychiatric Association Press.

Bilgel, M., An, Y., Lang, A., Prince, J., Ferrucci, L., Jedynak, B., \& Resnick, S.M. (2014). Trajectories of Alzheimer disease-related cognitive measures in a longitudinal sample. Alzheimers Dement, 10(6), 735-742.e734. doi: 10.1016/j.jalz.2014.04.520

Blessed, G., Tomlinson, B.E., \& Roth, M. (1968). The association between quantitative measures and senile changes in the cerebral gray matter of elderly subjects. British Journal of Psychiatry, 114, 97-811.

Burnham, K.P. \& Anderson, D.R. (2002). Model selection and multimodel inference: A practical information-theoretic approach (2nd ed.). New York: Springer-Verlag.

Buschke, H. (1984). Cued recall in Amnesia. Journal of Clinical and Experimental Neuropsychology, 6(4), 433-440.

Chang, Y.-L., Bondi, M.W., Fennema-Notestine, C., McEvoy, L.K., Hagler Jr, D.J., Jacobson, M.W., \& Dale, A.M. (2010). Brain substrates of learning and retention in mild cognitive impairment diagnosis and progression to Alzheimer's disease. Neuropsychologia, 48(5), 1237-1247. doi: 10.1016/j.neuropsychologia.2009.12.024

Cloutier, S., Chertkow, H., Kergoat, M.J., Gauthier, S., \& Belleville, S. (2015). Patterns of cognitive decline prior to dementia in persons with mild cognitive impairment. Journal of Alzheimer's Disease, 47(4), 901-913. doi: 10.3233/jad-142910

Donohue, M.C., Sperling, R.A., Salmon, D.P., Rentz, D.M., Raman, R., Thomas, R.G., Weiner, M., \& Aisen, P.S. (2014). The preclinical alzheimer cognitive composite: Measuring amyloid-related decline. JAMA Neurology, 71(8), 961-970.

Grober, E. \& Buschke, H. (1987). Genuine memory deficits in dementia. Developmental Neuropsychology, 3, 13-36.

Grober, E. \& Kawas, C. (1997). Learning and retention in preclinical and early Alzheimer's Disease. Psychology and Aging, 12, $183-188$

Grober, E., Hall, C.B., Lipton, R.B., Zonderman, A.B., Resnick, S.M., \& Kawas, C. (2008). Memory impairment, executive dysfunction, and intellectual decline in preclinical Alzheimer's disease. Journal of the International Neuropsychological Society, 14(2), 266-278.

Grober, E., Veroff, A.E., \& Lipton, R.B. (2018). Temporal unfolding of declining episodic memory on the Free and Cued Selective Reminding Test in the predementia phase of Alzheimer's disease: Implications for clinical trials. Alzheimer's \& Dementia: Diagnosis, Assessment \& Disease Monitoring, 10, 161-171. doi:10.1016/j.dadm.2017.12.004 
Hall, C.B., Derby, C., LeValley, A., Katz, M.J., Verghese, J., \& Lipton, R.B. (2007). Education delays accelerated decline on a memory test in persons who develop dementia. Neurology, 69(17), 1657-1664. doi:10.1212/01.wnl.0000278163. 82636.30

Hall, C.B., Ying, J., Kuo, L., \& Lipton, R.B. (2003). Bayesian and profile likelihood change point methods for modeling cognitive function over time. Computational Statistics and Data Analysis 4, 91-109.

Hall, C.B., Ying, J., Kuo, L., Sliwinski, M., Buschke, H., Katz, M., \& Lipton, R.B. (2001). Estimation of bivariate measurements having different change points, with application to cognitive aging. Statistics in Medicine, 20(24), 3695-3714.

Howieson, D., Carlson, N., Moore, M., Wasserman, D., Abendroth, C., Payne-Murphy, J., \& Kaye, J. (2008). Trajectory of mild cognitive impairment onset. Journal of the International Neuropsychological Society, 14(2), 192-198. doi: 10.1017/S135 5617708080375

McKhann, G., Drachman, D., Folstein, M., Katzman, R., Price, D., \& Stadlan, E.M. (1984). Clinical diagnosis of Alzheimer's Disease: Report of the NINCDS-ADRA work group under the auspices of Department of Health and Human Services Task force on Alzheimer's Disease. Neurology, 34, 939-944.

Mistridis, P., Krumm, S., Monsch, A.U., Berres, M., \& Taylor, K.I. (2015). The 12 years preceding mild cognitive impairment due to Alzheimer's disease: the temporal emergence of cognitive decline. Journal of Alzheimer's Disease, 48(4), 1095-1107. doi: 10.3233/jad-150137

Mormino, E.C., Papp, K.V., Rentz, D.M., Donohue, M.C., Amariglio, R., Quiroz, Y.T., Chhatwal, J., Marshall, G.A., Donovan, N., Jackson, J., \& Gatchel, J.R. (2017). Early and late change on the preclinical Alzheimer's cognitive composite in clinically normal older individuals with elevated beta-amyloid. Alzheimers Dementia, 13(9), 1004-1012.

Papp, K.V., Rentz, D.M., Mormino, E.C., Schultz, A.P., Amariglio, R.E., Quiroz, Y., Johnson, K.A., \& Sperling, R.A. (2017). Cued memory decline in biomarker-defined preclinical Alzheimer disease. Neurology, 88(15), 1431-1438.

Schindler, S.E., Jasielec, M.S., Weng, H, Hassenstab, J.J., Grober, E., McCue, L.M., Morris, J.C., Holtzman, D.M., Xiong, C., \& Fagan, A.M. (2017). Neuropsychological measures that detect early impairment and decline in preclinical Alzheimer disease. Neurobiol Aging, 56, 25-32.

Soldan, A., Pettigrew, C., Cai, Q., Wang, J., Wang, M.-C., Moghekar, A., Miller, M.I., Albert, M., \& BIOCARD Research Team. (2017). Cognitive reserve and long-term change in cognition in aging and preclinical Alzheimer's disease. Neurobiology of Aging, 60, 164-172. doi:10.1016/j.neurobiolaging.2017. 09.002

Stern, Y. (2002). What is cognitive reserve? Theory and research application of the reserve concept. Journal of the International Neuropsychol Society, 8, 448-460.

Wagner, M., Wolf, S., Reischies, F.M., Daerr, M., Wolfsgruber, S., Jessen, F., Popp, J., Maier, W., Hüll, M., Frölich, L., Hampel, H., Perneczky, R., Peters, O., Jahn, H., Luckhaus, C., Gertz, H.J., Schröder, J., Pantel, J., Lewczuk, P., Kornhuber, J., \& Wiltfang, J. (2012). Biomarker validation of a cued recall memory deficit in prodromal Alzheimer disease. Neurology, 78(6), 379-386. doi: 10.1212/WNL.0b013e318245f447

Welsh, K., Butters, N., Hughes, J., Mohs, R., \& Heyman, A. (1991). Detection of abnormal memory decline in mild cases of Alzheimer's disease using CERAD neuropsychological measures. Archives of Neurology, 48(3), 278-281. doi: 10.1001/ archneur.48.3.278 